\title{
Latent trait modeling of tau neuropathology in progressive supranuclear palsy
}

\author{
Naomi Kouri ${ }^{1}$ - Melissa E. Murray ${ }^{1} \cdot J^{\prime}$ Joseph S. Reddy ${ }^{2}$. Daniel J. Serie ${ }^{2} \cdot$ Alexandra Soto-Beasley $^{1} \cdot$ Mariet Allen $^{1}$. \\ Minerva M. Carrasquillo ${ }^{1}$. Xue Wang ${ }^{1,2}$. Monica Casey Castanedes ${ }^{1} \cdot$ Matthew C. Baker $^{1} \cdot$ Rosa Rademakers $^{1,3}$. \\ Ryan J. Uitti ${ }^{4}$. Neill R. Graff-Radford ${ }^{4}$. Zbigniew K. Wszolek ${ }^{4}$. Gerard D. Schellenberg ${ }^{5}$. Julia E. Crook ${ }^{2}$. \\ Nilüfer Ertekin-Taner ${ }^{1,4} \cdot$ Owen A. Ross $^{1} \cdot$ Dennis W. Dickson ${ }^{1}$ (I)
}

Received: 23 November 2020 / Revised: 15 February 2021 / Accepted: 16 February 2021 / Published online: 26 February 2021

(c) The Author(s) 2021

\begin{abstract}
Progressive supranuclear palsy (PSP) is the second most common neurodegenerative Parkinsonian disorder after Parkinson's disease, and is characterized as a primary tauopathy. Leveraging the considerable clinical and neuropathologic heterogeneity associated with PSP, we measured tau neuropathology as quantitative traits to perform a genome-wide association study (GWAS) within PSP to identify genes and biological pathways that underlie the PSP disease process. In 882 PSP cases, semi-quantitative scores for phosphorylated tau-immunoreactive coiled bodies (CBs), neurofibrillary tangles (NFTs), tufted astrocytes (TAs), and tau threads were documented from 18 brain regions, and converted to latent trait (LT) variables using the R ltm package. LT analysis utilizes a multivariate regression model that links categorical responses to unobserved covariates allowing for a reduction of dimensionality, generating a single, continuous variable to account for the multiple lesions and brain regions assessed. We first tested for association with PSP LTs and the top PSP GWAS susceptibility loci. Significant SNP/LT associations were identified at rs242557 (MAPT H1c sub-haplotype) with hindbrain CBs and rs 1768208 $(M O B P)$ with forebrain tau threads. Digital microscopy was employed to quantify phosphorylated tau burden in midbrain tectum and red nucleus in 795 PSP cases and tau burdens were used as quantitative phenotypes in GWAS. Top associations were identified at rs1768208 with midbrain tectum and red nucleus tau burden. Additionally, we performed a PSP LT GWAS on an initial cohort, a follow-up SNP panel (37 SNPs, $P<10^{-5}$ ) in an extended cohort, and a combined analysis. Top SNP/LT associations were identified at SNPs in or near SPTBN5/EHD4, SEC13/ATP2B2, EPHB1/PPP2R3A, TBC1D8, IFNGR1/OLIG3, ST6GAL1, HK1, CALB1, and SGCZ. Finally, testing for SNP/transcript associations using whole transcriptome and whole genome data identified significant expression quantitative trait loci at rs3088159/SPTBN5/EHD4 and rs 154239/GHRL. Modeling tau neuropathology heterogeneity using LTs as quantitative phenotypes in a GWAS may provide substantial insight into biological pathways involved in PSP by affecting regional tau burden.
\end{abstract}

Keywords Progressive supranuclear palsy $\cdot M A P T \cdot M O B P \cdot$ Tau $\cdot$ Latent traits

Dennis W. Dickson

dickson.dennis@mayo.edu

1 Department of Neuroscience, Mayo Clinic, 4500 San Pablo Road, Jacksonville, FL 32224, USA

2 Department of Health Sciences Research, Mayo Clinic, Jacksonville, FL, USA

3 VIB-UAntwerp Center for Molecular Neurology, Antwerp, Belgium

4 Department of Neurology, Mayo Clinic, Jacksonville, FL, USA

5 Department of Pathology and Laboratory Medicine, University of Pennsylvania, Philadelphia, PA, USA

\section{Introduction}

Progressive supranuclear palsy (PSP) is an atypical Parkinsonian disorder where patients typically exhibit early unexplained falls, vertical gaze palsy, axial rigidity, and levodopa unresponsive parkinsonism. PSP is a primary tauopathy because on neuropathologic examination there is predominately hyperphosphorylated, aggregated tau protein. Microtubule associated protein tau encoded by the MAPT gene binds to microtubules and is important for maintaining neuronal morphology and function. 
PSP neuropathologic features include tau-immunoreactive neuronal and glial lesions in the basal ganglia, diencephalon, and brainstem with variable involvement of the neocortex. Macroscopic examination in PSP reveals pigment loss in the substantia nigra and atrophy of multiple brain regions including superior cerebellar peduncle, subthalamic nucleus, hilus of the cerebellar dentate, and midbrain with dilation of the aqueduct of Sylvius. Neuropathologic diagnostic criteria for PSP require the presence of tau neurofibrillary tangles in the most affected nuclei which are the globus pallidus, subthalamic nucleus, and substantia nigra [19, 31]. Tufted astrocytes (TA) are astrocytic tau lesion found consistently in the motor cortex and striatum in PSP [34, 38] and oligodendroglial coiled bodies (CB), often accompanied by neuropil threads in the white matter of the diencephalon, brainstem, and cerebellum [5].

Aside from the typical PSP profile, there exists neuropathologic variants of PSP [14]. Reflecting the heterogeneity of PSP tau pathology distribution, PSP patients can present with a variety of clinical presentation including frontotemporal dementia, spastic paraparesis, progressive apraxia if speech, corticobasal syndrome, primary gait failure with freezing, and a Parkinsonian-like syndrome that is initially responsive to levodopa therapy $[1,51]$. In patients with primary gait failure with freezing there is greater tau pathology in the globus pallidus, diencephalon, and brainstem and more specifically, pallido-nigro-luysial atrophy with very mild if any cortical pathology. PSP presenting with typical Parkinson's disease features may have milder tau pathology overall.

Although there are rare familial PSP cases [16] and an estimated $10-15 \%$ positive family history of neurological disorder, PSP is considered a sporadic disorder. Despite the majority of PSP patients reported to have negative family history, there is a strong genetic risk factor located on chromosome $17 \mathrm{q} 21$ composed of a $\sim 1.2 \mathrm{Mb}$ inversion surrounding MAPT termed the $\mathrm{H} 1$ and $\mathrm{H} 2$ haplotypes [6, 12]. The MAPT locus associated with PSP risk was further analyzed and identified subhaplotypes in the H1 haplotype background that associate with risk of developing disease $[40,43]$. However, the H1 haplotype frequency is $~ 80 \%$ in healthy control populations of European ancestry [15] and therefore alone, $\mathrm{H} 1$ is not sufficient to cause disease. The H1 and H1c subhaplotype association with PSP was confirmed and further strengthened in the largest PSP genetic association study to date [20]. Additionally, five novel PSP susceptibility loci have been identified at MOBP, STX6, EIAF2AK3, SLCO1A2I, and DUSP10 [11, 46]. These new susceptibility loci identified in the PSP GWASs have the potential to reveal new insights into PSP, but need to be further studied to understand their role in disease pathogenesis.

Latent variable models are based on Item Response Theory (IRT) and are commonly used to link latent traits (LT) to unobserved covariates using dichotomous or polytomous manifest variables [45]. This allows one to draw conclusions from complex datasets and summarize the information by a reduction of dimensionality, often applied to psychometric testing and tests that measure personality traits, cognitive traits, moods, and behavioral dispositions. Semejima's graded response model (GRM) is an extension of the basic IRT model, which analyzes polytomous rather than dichotomous responses. Here we apply the GRM to semi-quantitative tau neuropathology (i.e. polytomous variables) to generate a LT variable summarizing the tau neuropathology profile for each individual PSP case using the ltm R package [44]. This approach to reducing the complex dataset of four tau lesions in 18 brain regions per PSP case allows for quantitative trait testing in genetic association studies.

\section{Methods}

\section{Neuropathologic evaluation}

Neuropathologically-confirmed PSP cases from the Mayo Clinic Florida brain bank were evaluated by one neuropathologist (Dennis W. Dickson) and selected for inclusion in this study based on the completeness of tau neuropathology data and availability of frozen tissue for DNA extraction. Sixteen sections from one formalin-fixed hemisphere were systematically sampled, paraffin embedded, and cut into $5 \mu \mathrm{m}$ slices. Tissue sections were deparaffinized then pretreated with steam in deionized water for $30 \mathrm{~min}$ and stained with anti-phosphorylated tau CP13 (1:1000, pSer202, kind gift from Dr. Peter Davies, Feinstein Institutes for Medical Research), or pretreated with steam in tris $\mathrm{pH}=9$ buffer for $30 \mathrm{~min}$ and stained with anti-MOBP (1:250, Thermo Fisher Scientific cat\# PA5-72493) using a Lab Vision Autostainer 480S (Thermo Fisher Scientific, Waltham, MA, USA). Immunostained slides were subsequently counterstained with hematoxylin. All postmortem samples were acquired with appropriate ethical approval and this study was approved by the Mayo Clinic Institutional Review Board.

Semiquantitative tau pathology measures (none $=0$, mild $=1$, moderate $=2$, severe $=3$ ) were assessed from phosphorylated tau immunostained sections in 18 different anatomical structures for the following lesions: NFTs, CBs, TAs, and threads. Brain regions were selected based on those affected in PSP, which include: basal nucleus, caudate putamen, globus pallidus, hypothalamus, motor cortex, subthalamic nucleus, thalamic fasciculus, ventral thalamus, cerebellar white matter, dentate nucleus, inferior olive, locus ceruleus, medullary tegmentum, midbrain tectum, oculomotor complex, pontine base, pontine tegmentum, red nucleus, and substantia nigra. Temporal cortex tau pathology scores 
were also collected but excluded from further analyses because the distribution of scores for the sample set was skewed and incomplete due to this region being minimally affected in PSP.

\section{Latent variable modeling}

Polytomous ordinal data was handled using the Graded Response Model (GRM) within the ltm R package [44]. Semiquantitative tau pathology data was available for 906 patients and were used to generate LTs although some were later excluded from analyses based on genome-wide genotyping quality control. The 72 total pathology measures (18 brain regions and 4 tau lesions per region) were each on a 0 to 3 scale and were used to create an overall score for degree of pathology based on a latent trait approach. Temporal cortex exhibited highly skewed or bimodal distributions and were therefore excluded from LT calculations. The overall latent variable score is an estimate of an assumed underlying level of pathology severity that all individual scores are dependent on or correlated with. Brain regions were divided into "forebrain" and "hindbrain" categories defined by the mesencephalic/diencephalic junction. Twelve LT measures were used as intermediate phenotypes and these included $\mathrm{CB}$ overall, CB hindbrain, CB forebrain, NFT overall, NFT hindbrain, NFT forebrain, TA overall, TA hindbrain, TA forebrain, tau threads overall, tau threads hindbrain, tau threads forebrain, Three additional LT measures were generated for overall, forebrain, and hindbrain LTs, which included all four lesions for all brain regions and regions above and below the mesencephalic/diencephalic junction. Information curves are a feature generated as part of the ltm package and illustrate the information provided by each brain region as the area under the curve used to calculate LTs.

\section{Digital neuropathology}

IHC slides were scanned at $20 \mathrm{X}$ on an Aperio AT2 digital whole slide scanner (Leica Biosystems, Buffalo Grove, IL, USA), which converts slides into high-resolution digital images. Regions of interest were selected based on the overall LT information curve in order to minimize total number of regions analyzed. Midbrain tectum and red nucleus were analyzed at the level of the $3^{\text {rd }}$ cranial nerve on midbrain sections. ImageScope v12.4.2 (Leica Biosystems) software was used to trace regions of interest as annotation layers. A custom color deconvolution macro (v9) was used to analyze CP13 immunoreactivity by detecting only $\mathrm{DAB}+$ color profile as a percent strong threshold while calculating all other colors as negative (percent medium and weak thresholds) (Online Resource Fig. 2). Regions of interest on MOBPimmunostained slides from superior frontal cortex and pons were annotated similarly and included corpus callosum, anterior cingulate white matter, superior frontal white matter, deep frontal white matter, and pontine base. A custom IHC nuclear macro (v1) was created to quantify small MOBP-immunoreactive granules in white matter of representative forebrain and hindbrain regions. The macro is able to discriminate nuclei from MOBP granules based on color and size and expressed as a percent $3+$ nuclei in the macro (Online Resource File of macro parameters).

\section{Genotyping}

Genome-wide genotyping data were generated using Illumina $660 \mathrm{~W}$-Quad chips and raw intensity files were subjected to cluster analysis in GenomeStudio software (Illumina, San Diego, CA, USA). Quality control of genotyping data was performed at the individual level and then at the SNP level. For quality control, 10 individuals were genotyped in duplicate. Exclusion criteria for individual samples included high genotype failure rate $(>2 \%$ excluded 4 samples) and cryptic relatedness or sample duplicates (all samples met these criteria). Gender inconsistencies were assessed by chromosome X genotypes which excluded 29 individuals based on observed and expected gender. Exclusion criteria for markers included minor allele frequency (7420 SNPs were removed because of minor allele frequency $<0.1 \%)$ and high genotype failure rates $(33,191$ SNPs were removed because of genotype failure rates $>2 \%$ ).

Selection criteria for the extended PSP cohort followup SNPs (Online Resource Table 1) include LT/genotype associations with $P<10^{-5}(N=37)$ and that the SNPs were in Hardy-Weinberg equilibrium $(P<0.05)$. Based on these top loci, an additional $~ 550$ SNPs for each LT $(P<0.001)$ were further scrutinized by determining the linkage disequilibrium structure between the SNP clusters. Based on this, the most significant SNPs were included and those in tight linkage disequilibrium $\left(R^{2}>0.95\right)$ with these top SNPs were excluded from further analysis. Genotyping of Stage 2 SNPs was performed on the MassArray iPlex platform (Agena Bioscience, San Diego, CA, USA). Genetic variants that were not compatible with iPlex technology were analyzed by individual pre- and custom-designed Taqman assays following manufacturer protocol (Thermo Fisher Scientific, Waltham, MA, USA) or by direct sequencing analyses. Sanger sequencing was performed on PCR reactions. PCR products were purified using AMPure (Beckman Coulter, Brea, CA, USA) then sequenced in both directions using the BigDye Terminator cycle sequencing kit (Thermo Fisher Scientific, Waltham, MA, USA). Sequencing reactions were purified using CleanSEQ (Beckman Coulter, Brea, CA, USA) and analyzed on an ABI3730xl Genetic Analyzer. AMPure and CleanSEQ purifying reactions were performed on a Biomek FX Laboratory Automation Workstation (Beckman Coulter, Brea, CA, USA). Base calling, 
sequence alignments and heterozygote detection will be performed using Sequencher v4.8 (Gene Codes, Ann Arbor, MI, USA).

\section{Relatedness and population stratification}

Relationships among samples were evaluated using KINGrobust [33] in PLINK2 [10] (v2.00a3LM) using the "-makeking-table" flag. Samples related up to the 3rd degree (Kinship coefficient $\geq 0.0442$ ) were identified and one sample from each pair of relateds with the best call rate was retained. After resolving relatedness, the underlying population substructure was evaluated using Eigenstrat $[39,41]$ to identify and remove population outliers. Eigenstrat was set to remove outliers of up to 6 standard deviations of the top 10 principal components (PCs) over five iterations, while refitting PCs after each iteration of outlier removal. While one sample was removed for relatedness and six samples identified as population outliers were also removed.

\section{Imputation}

Samples and variants that passed QC were imputed to the Haplotype Reference Consortium (HRC) reference panel [35] using Minimac4 imputation algorithm [13] with Eagle phasing [32] implemented by the Michigan Imputation Server (MIS, v1.5.7) [13]. Prior to imputation, the strand and position of variants were harmonized with the HRC reference panel (r1.1 2016) using tools provided by the McCarthy Group at the University of Oxford, UK (https://www.well. ox.ac.uk/ wrayner/tools/index.html). Harmonized genotypes were exported to VCF files and uploaded to the MIS for imputation. Given that the imputation process replaces original genotypes with imputed ones, an in-house script was utilized to reinstate the original genotypes to the VCF after imputation. Variant dosages from imputation were imported into PLINK 2 (v2.00a3LM) using the "dosage = DS" flag. Variants with in imputation $R^{2} \geq 0.9$ and a minor allele frequency (MAF) $\geq 2 \%$ were retained for LT GWAS.

\section{Mayo RNAseq and WGS datasets}

The Mayo RNAseq dataset comprises transcriptome measures from temporal cortex (TCX) and cerebellum (CER); RNA isolation, data collection, sequencing alignment, counting and QC has been described in detail elsewhere [2, 4]. Gene counts were normalized using conditional quantile normalization (CQN) [18]. DNA sample processing and sequencing protocols are described on the AMP-AD knowledge portal: https://adknowledgeportal.synapse.org/, Synapse ID: syn10901601. FastQ files from sequencing were processed through Mayo Clinic's GenomeGPS pipeline. Briefly, reads were aligned to the reference (hg19) using Novoalign and variant calling and genotyping was performed while implementing GATK's Best Practices Workflow [48]. Samples were QC-ed for coverage (at least $90 \%$ covered at $10 \times$ and $50 \%$ covered at $30 \mathrm{x}$ ), genotyping quality (median GQ of 99), call rate (95\%), transition to transversion (Ti/Tv) ratio (between 2 and 2.1), sex (PLINK inbreeding coefficient of the X-chromosome for males $>0.7$ and females <0.3) and contamination (VerifyBamID [25] FREEMIX score less than 0.02). Subsequently, samples were evaluated for relatedness up to $3^{\text {rd }}$ degree, population substructure and sequencing batch effects. Variants passing VQSR filter, having a genotyping rate of $95 \%$ or more, a Bonferroni adjusted Hardy-Weinberg $p$ value greater than 0.05 in controls and BLAT [26] score less than four were retained for downstream analysis. All 349 samples and 19,357,792 variants passed QC. Genotypes were extracted from VCF files using PLINK [10]. CQN gene expression values and accompanying WGS genotypes of variants having a minor allele frequency equal to or greater than $5 \%$ in samples with expression data were subsequently utilized for eQTL analysis.

\section{Statistical analysis}

Variants within $\pm 1 \mathrm{Mb}$ of the Ensembl gene locus were tested for association with CQN gene expression levels (eQTL) using a linear mixed model implemented with the lme4 package [7] in $\mathrm{R}$ statistical software version 3.5.2. CQN expression value was the dependent variable; variant dosage $(0,1$ or 2$)$ was the independent variable. All QTL models were adjusted for diagnosis, sex, age at death, RIN, APOE $\varepsilon 4$ dosage, tissue source, flowcell and the first three principle components, with flowcell being the random effects variable. Denominator degrees of freedom for test statistic was obtained using Kenward-Roger [27] restricted maximum likelihood approximation in the lmerTest package [30] in R. False-discovery rate adjusted (Benjamini-Hochberg) q-values were calculated in R for all tested eQTLs.

For PSP LT GWAS study the initial PSP cohort $(n=498)$ with genome-wide genotyping data was tested for genetic association with LTs by linear regression under an additive model using age at death and sex as covariates with PLINK [42]. With $\alpha=0.05$ and Bonferroni correction for the 559,348 SNPs tested in stage 1 , a $P$ value of $9.0 \times 10^{-8}$ is required for 'genome-wide' significance. For the combined analysis, an extended PSP cohort was tested for association with PSP LTs by the same method as the primary PSP cohort, and followed by a combined analysis using PLINK. The PSP LT GWAS was re-analyzed using imputed genotypes in the initial PSP cohort $(n=472)$. Variant dosages were tested for their association with latent traits in PLINK2 (v2.00a3LM) using -glm flag using age at death, sex, and 
first four principle components to adjust for population substructure. Results were annotated using Annovar [49].

\section{Results}

\section{Latent trait association with top PSP susceptibility loci}

Semiquantitative tau pathology measures (none $=0$, mild $=1$, moderate $=2$, severe $=3$ ) were assessed from CP13 immunostained sections in 18 different anatomical structures for the following lesions: tufted astrocytes (TA) (Fig. 1a) oligodendroglial coiled bodies (CB) (Fig. 1b), neurofibrillary tangles (NFT) (Fig. 1c), and tau neuropil threads (Fig. 1d). PSP LTs were then generated from these semi-quantitative tau pathology scores for the study cohort $(N=904$, Table 1$)$ using the R ltm package. LTs were generated for each tau lesion type, separated into hindbrain and forebrain regions, and an overall LT variable i.e. all brain regions per lesion. Plotting semi-quantitative scores against LTs, shows that the original information is not lost, as there is an increase in LT as semi-quantitative score increase (Fig. 2a). This resulted in a total of 16 LT phenotypes tested for genetic association. As part of the ltm $\mathrm{R}$ package, Information plots are generated with the LT scores in order to display the contribution of information provided to the LT score per item (i.e. anatomical structures). The Overall Item Information Curve indicates that the items locus ceruleus and temporal cortex provide little information in the whole latent trait continuum (Fig. 2b). The greatest information is provided by the items midbrain tectum, red nucleus, pontine base, ventral thalamus, and motor cortex which is consistent with what we observe in PSP tau neuropathology. For example,
Fig. 1 Tau-immunoreactive lesions in PSP. Characteristic phosphorylated tau-immunoreactive lesions in PSP were used to generate latent trait scores from semi-quantitative scores using the $\mathrm{ltm} \mathrm{R}$ package. Tufted astrocytes (a), oligodendroglial coiled bodies (b), neurofibrillary tangles (c), and tau neuropil threads (d). Scale bar $=30 \mu \mathrm{m}$
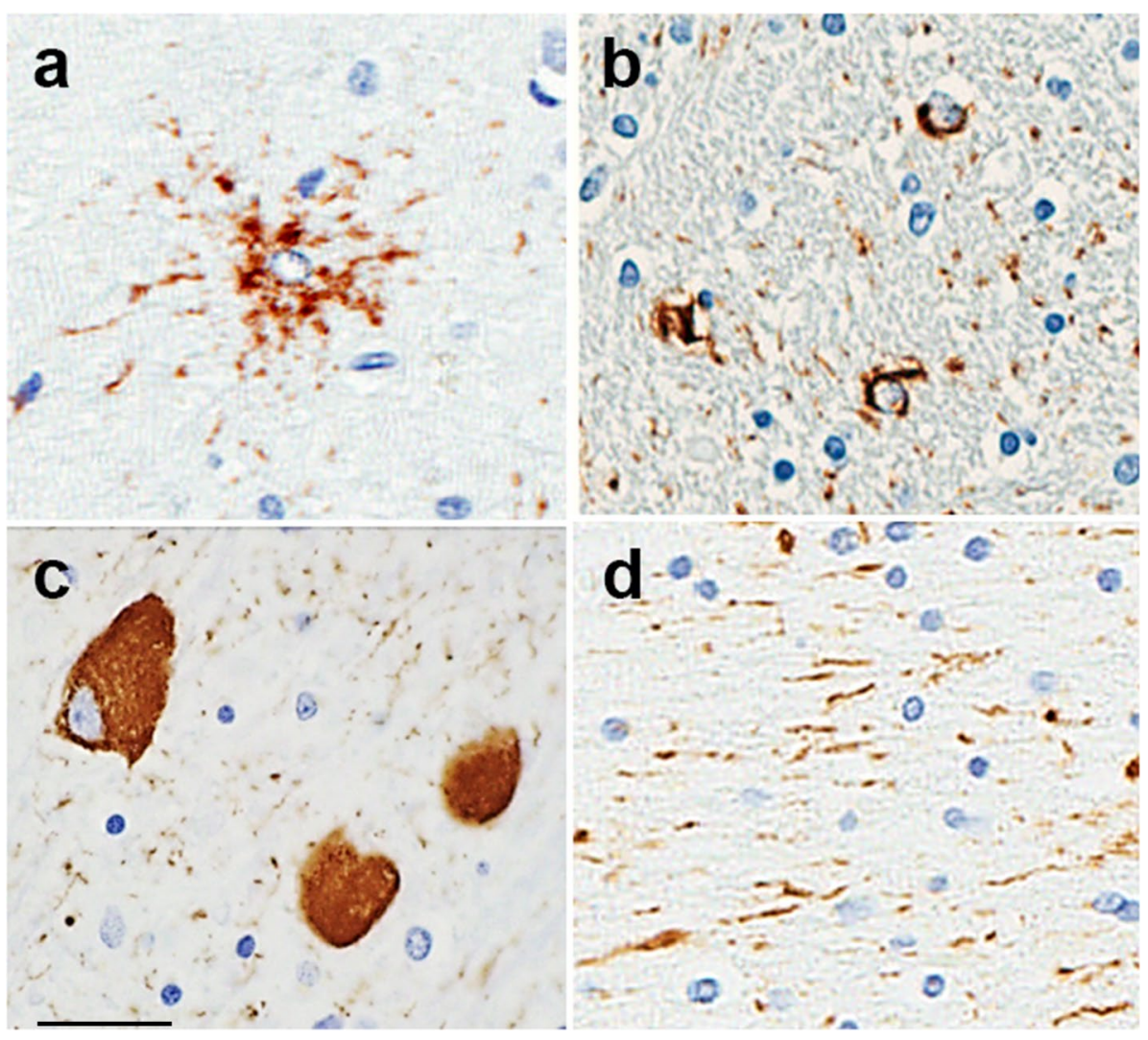

Table 1 Demographics of PSP cases

\begin{tabular}{lllllll}
\hline PSP Cohort & $\begin{array}{l}\text { Samples ana- } \\
\text { lyzed }\end{array}$ & M: F & Female $\%$ & \multicolumn{2}{l}{ Age at death (y) } \\
\cline { 5 - 7 } & & & & Mean age & Range & s.d \\
\hline Initial & 481 & $245: 235$ & 49 & 75.0 & $46-98$ & 8.0 \\
Extended & 401 & $233: 168$ & 43 & 74.7 & $45-91$ & 7.5 \\
Combined & 882 & $478: 403$ & 46 & 74.9 & $45-98$ & 7.8 \\
\hline
\end{tabular}

$M$, male; $F$, female; s.d., standard deviation 


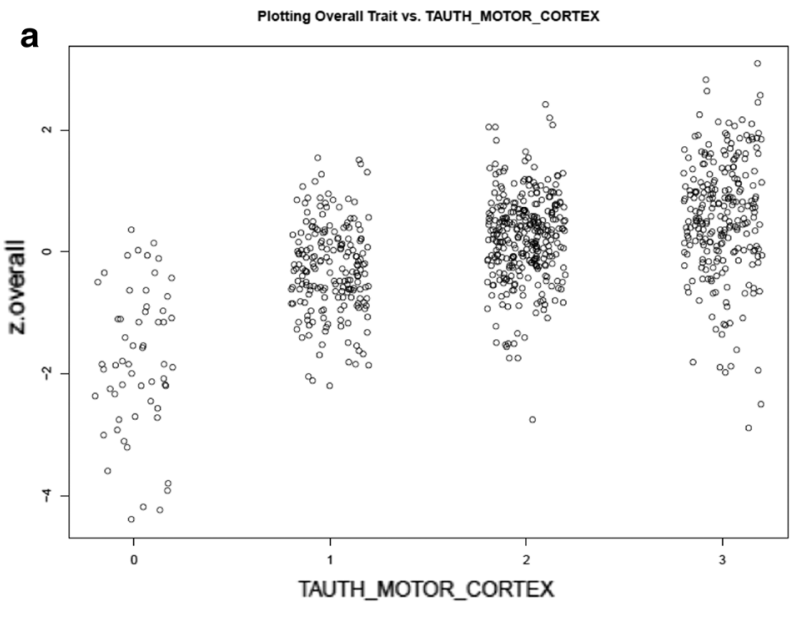

Fig. 2 Latent variable modeling of PSP neuropathology. This representative scatter plot shows the relationship of latent traits to semiquantitative tau pathology scores. Information from the original data is not lost as there is an increase in the overall LT score as semiquantitative score increase for tau threads in the motor cortex (a). The $\mathrm{ltm} \mathrm{R}$ package generates Information plots in order to understand the

tau pathology burden in locus ceruleus and temporal cortex are not highly variable, whereas the midbrain structures and motor cortex have considerable heterogeneity in affection, hence the hindbrain- and forebrain-predominant PSP subtypes. Information Curve plots for NFTs, CBs, TAs, and Threads show variability in item information per lesion type (Online Resource Fig. 1).

PSP LTs were then used as quantitative traits to test for genetic association using linear regression under an additive model using age and sex as covariates. We tested for association with the top PSP susceptibility loci at MAPT (rs8070723 for the H1H2 haplotype; rs242557 for H1c haplotype), MOBP (rs1768208), EIF2AK3 (rs7571971), and STX6 (rs1411478), SLCO1A2 (rs11568563), and DUSP10 (rs6687758) [11, 20, 46]. Meta-analysis of these five SNPs resulted in significant associations with rs242557 and LTs measuring glial tau lesion load (Table 2). The regional effect of genotype on tau pathology burden in PSP can be visualized upon stratification by rs 242557 genotype where "A" is the risk allele and " $G$ " the protective allele (Fig. 3). The $\mathrm{H} 1 \mathrm{c}$ haplotype appears to have regional effects on tau burden in PSP. Each copy of the "A" risk allele is associated with less TA burden in forebrain structures $\left(P=1.56 \times 10^{-4}\right.$, Beta $=-0.159)$ (Fig. 3a), yet greater TA burden in hindbrain structures $\left(P=2.18 \times 10^{-3}\right.$, Beta $\left.=0.121\right)$ (Fig. 3b). Whereas the rs242557 "A" allele is associated with decreased CB load in both forebrain $\left(P=8.98 \times 10^{-2}\right.$, Beta $\left.=-0.066\right)($ Fig. 3c $)$ and hindbrain $\left(P=1.03 \times 10^{-6}\right.$, Beta $\left.=-0.201\right)$ (Fig. 3d).

Testing for association with PSP LTs at the MOBP locus (rs1768208) identified a significant LT/SNP association with rs1768208 and forebrain threads $\left(P=2.14 \times 10^{-5}\right.$,

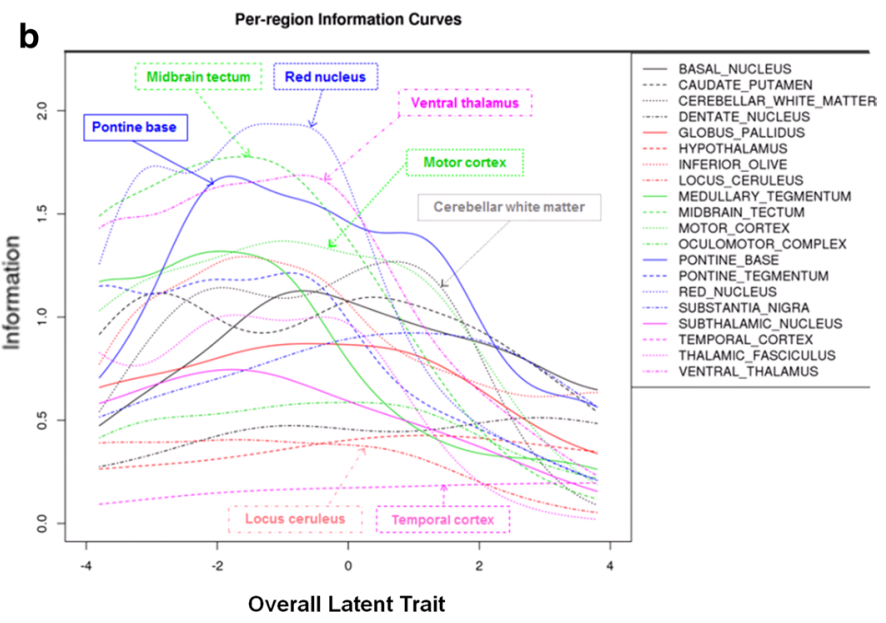

contribution of information provided to the LT score per item (i.e. anatomical structures). The Overall Item Information Curve indicates that the items locus ceruleus and temporal cortex provide little information in the whole latent trait continuum, whereas midbrain structures and motor cortex provide the greatest amount of information (b)

Beta $=0.169)($ Fig. $4 \mathrm{a})$, overall threads $\left(P=4.65 \times 10^{-4}\right.$, Beta $=0.145)$, and forebrain CBs $\left(P=5.16 \times 10^{-3}\right.$, Beta $=0.108)($ Table 2). These top associations show that rs 1768208/LT is associated with increased tau thread load in hindbrain and forebrain with each copy of the "C" minor allele (Fig. 4a, b). The remaining PSP susceptibility loci at MAPT ( $\mathrm{H} 1 \mathrm{H} 2$ haplotype-tagging SNP), STX6, EIF2AK3, SLCO1A2, and DUSP10 showed suggestive associations with LTs in PSP (Online Resource Table 2).

\section{Genome-wide association analysis with PSP tau and MOBP burden}

Regions of interest were selected from the overall LT information plot based on the area under the curve per brain region (Fig. 2b). The midbrain tectum and red nucleus were sampled at the level of the oculomotor nerve (Online Resource Fig. 2). Slides with CP13 immunohistochemistry were scanned to high-resolution digital images, midbrain tectum and red nucleus were annotated, and annotation were subjected to a custom color deconvolution macro to quantify a percent tau burden. A subset of PSP cases were selected from the initial cohort based on the availability of CP13 slides. Linear regression was employed to test for association between genome-wide genotyping using age and sex as covariates. SNPs that overlapped with PSP LT results were further tested for association in the stage 2 cohort of PSP cases with CP13 slides available $(N=375)$, and meta-analysis was performed (Table 3). Interestingly, the top SNP common across LT and tau QT associations was rs1768208 at MOBP. Full summary statistics for stage 1 PSP QT GWAS 
Table 2 Latent trait association result with MAPT H1c haplotype-tagging SNP rs242557 and MOBP SNP rs1768208 in a combined analysis of the initial and extended PSP cohorts

\begin{tabular}{|c|c|c|c|c|c|c|}
\hline \multicolumn{7}{|l|}{ rs 242557} \\
\hline \multirow[t]{2}{*}{ Latent trait } & \multicolumn{2}{|l|}{ Initial } & \multicolumn{2}{|c|}{ Extended } & \multicolumn{2}{|c|}{ Combined } \\
\hline & BETA & $P$ value & BETA & $P$ value & BETA & $P$ value \\
\hline CB hind & -0.196 & $3.06 \times 10^{-4}$ & -0.207 & $1.20 \times 10^{-3}$ & -0.201 & $1.03 \times 10^{-6}$ \\
\hline CB overall & -0.163 & $2.31 \times 10^{-3}$ & -0.154 & $2.44 \times 10^{-2}$ & -0.160 & $1.42 \times 10^{-4}$ \\
\hline TA fore & -0.222 & $6.43 \times 10^{-5}$ & -0.071 & 0.28 & -0.159 & $1.56 \times 10^{-4}$ \\
\hline TA overall & -0.208 & $2.92 \times 10^{-4}$ & -0.089 & 0.20 & -0.160 & $2.82 \times 10^{-4}$ \\
\hline TA hind & 0.145 & $4.77 \times 10^{-3}$ & 0.086 & 0.17 & 0.121 & $2.18 \times 10^{-3}$ \\
\hline Hind overall & -0.120 & $3.16 \times 10^{-2}$ & -0.117 & $7.97 \times 10^{-2}$ & -0.119 & $5.42 \times 10^{-3}$ \\
\hline \multicolumn{7}{|l|}{ rs1768208 } \\
\hline \multirow[t]{2}{*}{ Latent trait } & \multicolumn{2}{|l|}{ Initial } & \multicolumn{2}{|c|}{ Extended } & \multicolumn{2}{|c|}{ Combined } \\
\hline & BETA & $P$ value & BETA & $P$ value & BETA & $P$ value \\
\hline Threads fore & 0.178 & $9.68 \times 10^{-4}$ & 0.157 & $8.00 \times 10^{-3}$ & 0.169 & $2.14 \times 10^{-5}$ \\
\hline Threads overall & 0.176 & $1.44 \times 10^{-3}$ & 0.104 & $9.92 \times 10^{-2}$ & 0.145 & $4.65 \times 10^{-4}$ \\
\hline Fore overall & 0.148 & $6.57 \times 10^{-3}$ & 0.092 & $1.89 \times 10^{-1}$ & 0.127 & $3.05 \times 10^{-3}$ \\
\hline $\mathrm{CB}$ fore & 0.143 & $3.51 \times 10^{-3}$ & 0.049 & $4.35 \times 10^{-1}$ & 0.108 & $5.16 \times 10^{-3}$ \\
\hline
\end{tabular}

$C B$, coiled bodies; $T A$, tufted astrocyte; $N F T$, neurofibrillary tangle; fore, forebrain structures; hind, hindbrain structures

Fig. 3 Latent trait scores stratified by rs 242557 genotypes indicate an association with glial tau burden. The rs 242557 MAPT H1c haplotype-tagging SNP is associated with variability in CB and TA burden. LT measures were generated from semi-quantitative scores of tau-immunoreactive CBs and TAs in a large PSP cohort and plotted stratified by rs 242557 genotype. These LT measures were used as phenotypes in linear regression analysis. The " $\mathrm{A}$ " risk allele is associated with decreased TA burden in the forebrain (a), increased TA burden in the hindbrain (b), and decreased forebrain (c) and hindbrain (d) CB burden. $L T$, latent trait; $C B$, coiled bodies; $T A$, tufted astrocytes; Blue bars represent the median
TA Forebrain

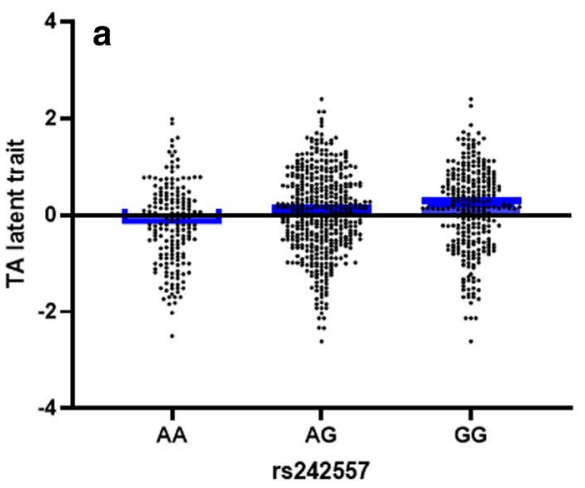

TA Hindbrain

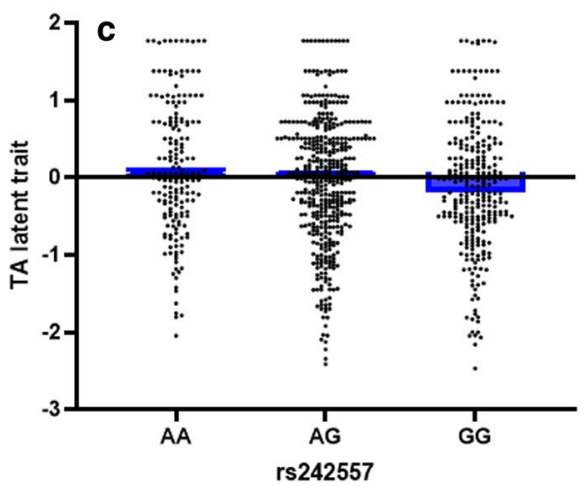

CB Forebrain

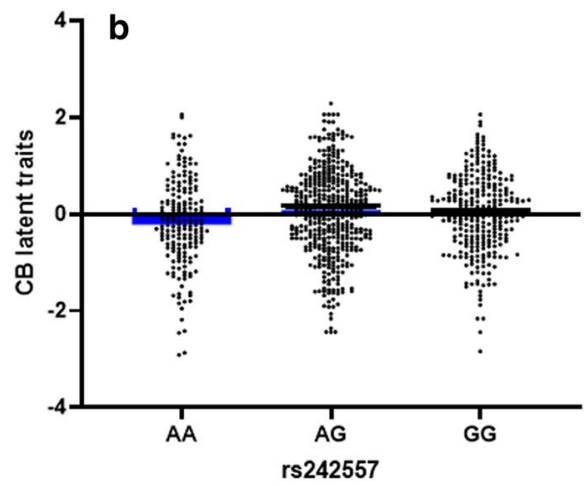

CB Hindbrain

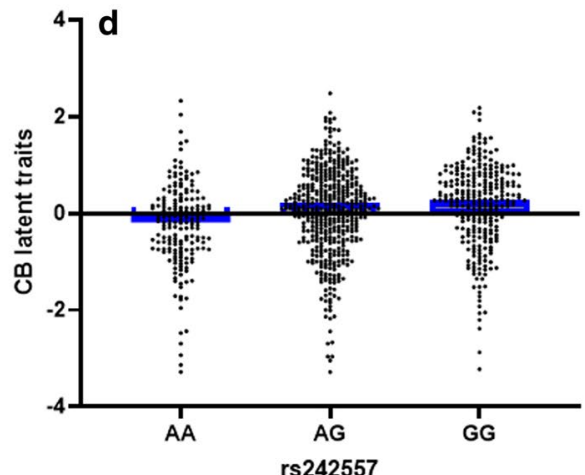




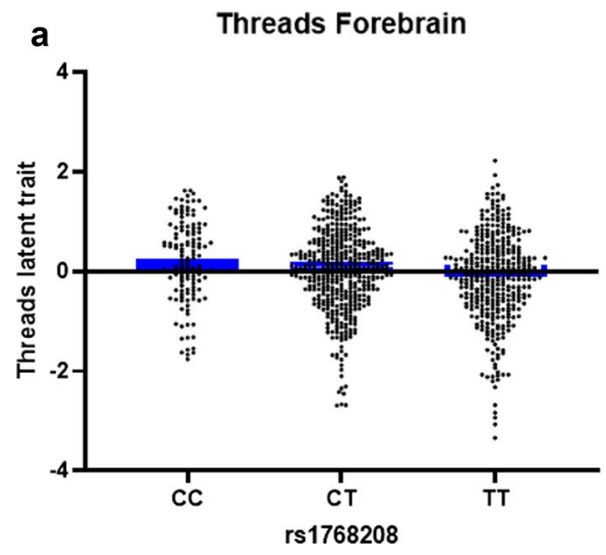

Fig. 4 Latent trait scores stratified by rs1768208 genotypes. LT measures were generated from semi-quantitative scores of tau-immunoreactive threads in a large PSP cohort and plotted stratified by rs1768208. Each data point represents the LT score for a PSP case. These LT measures were used as phenotypes in linear regression

of midbrain tectum and red nucleus for SNPs $P<10^{-3}$ are available in Online Resource Tables 3 and 4.

Since $M A P T$ and $M O B P$ genotypes are associated with tau pathology heterogeneity, we next sought to determine whether there is a genotype effect on MOBP pathology. MOBP is a component of the compact myelin sheath, is highly expressed in CNS white matter, and forms small granules in white matter of PSP (Online Resource Fig. 3). We selected superior frontal cortex to represent forebrain and pons to represent hindbrain. Digital image analysis to quantify MOBP granules in frontal cortex and pontine base did not detect a significant difference in MOBP granules across the various rs1768208 genotypes (Fig. 5).

\section{Genome-wide association analysis with PSP latent traits}

The initial PSP cohort $(N=481)$ with genome-wide genotyping and tau pathology LTs, were tested for LT/SNP associations using linear regression under an additive model and age and sex as covariates. This identified 62 SNPs associated with PSP LTs $\left(P<10^{-5}\right)$. Each quantitative trait locus identified was further scrutinized for LD structure, and of the 62 SNPs, 37 were selected to represent these top loci for further genotyping in an extended PSP cohort $(N=401)$. These analyses were also analyzed by linear regression using an additive model and age and sex as covariates, and then a combined analysis was performed in PLINK. Top SNP/ LT associations (Table 4) were identified at rs3088159 (located in intron 4 of $R P 11-23 P 13.6$, a non-coding RNA between $S P T B N 5 / E H D 4)$ with overall NFTs $\left(P=1.26 \times 10^{-6}\right.$, Beta $=-0.221)$, rs2305196 (HKl) with hindbrain threads $\left(P=2.72 \times 10^{-6}\right.$, Beta $\left.=-0.198\right)$, rs $154239($ SEC13) with

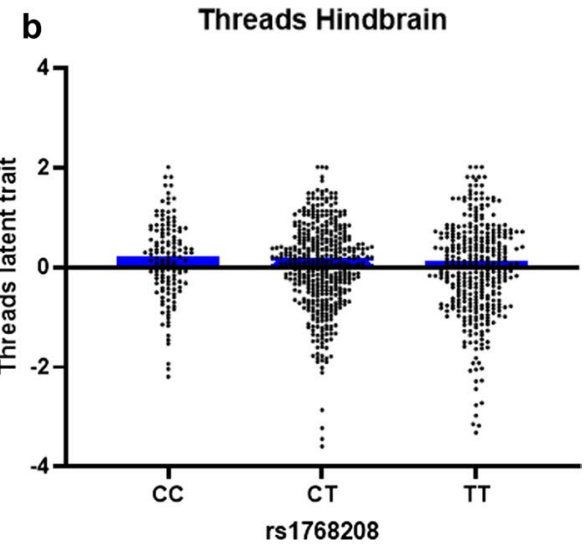

analysis. The top PSP GWAS SNP, rs17682208, is associated with tau thread pathology variability The "C" risk allele is associated with a decrease in tau thread burden compared to the " $\mathrm{T}$ " major allele in the forebrain (a) and hindbrain (b) in PSP. LT, latent trait; Blue bars represent the median

overall threads, and rs6543017 (TBC1D8) with forebrain CBs $\left(P=6.66 \times 10^{-6}\right.$, Beta $\left.=-0.252\right)$. These SNP/LT associations do not pass the Bonferroni correction for multiple testing. The complete results are presented in Online Resource Table 5. The most significant SNP/LT associations were identified at genetic loci that have not been previously implicated in PSP, but could potentially be biologicallyrelated to genes known to be involved in PSP pathogenesis based on PANTHER protein family/function analysis (Online Resource Table 6) [36].

Subsequently, we performed genetic imputation against HRC panel for variants with MAF $>2 \%$ and $\mathrm{R}^{2} \geq 0.90$, which resulted in $\sim 6$ million variants. Imputed genotypes were tested for genetic association with PSP LTs by linear regression under an additive model using age at death, sex, and the first four principle components for population substructure as covariates. This identified SNP/LT associations largely overlapping with previous results with the exception of one, rs2294892, which was significantly associated with hindbrain tau burden $\left(P<9 \times 10^{-8}\right)$. The SNP rs2294892 was then genotyped in 400 PSP cases comprising stage 2 cohort and meta-analysis was performed. The imputed SNP rs2294892 was nominally associated with hindbrain tau $\left(P=1.31 \times 10^{-5}\right)$.

\section{Brain transcriptome analysis}

We next sought to determine whether the 10 top PSP LT GWAS SNPs regulate expression of nearby genes. SNP/ transcript associations were tested on 349 Alzheimer's disease, PSP, and pathological aging temporal cortex and cerebellum RNA samples as previously described [2, 4]. SNPs within $\pm 1 \mathrm{Mb}$ of the Ensembl gene locus were 


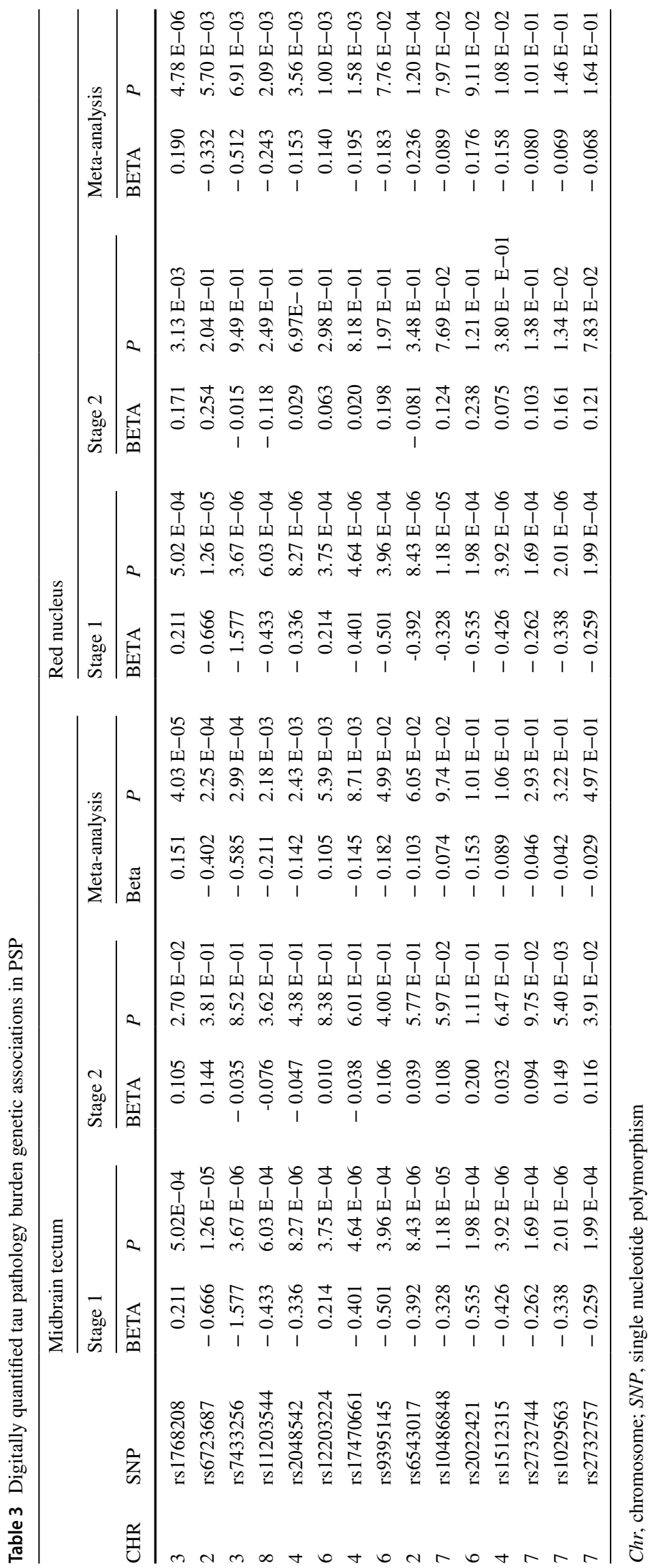



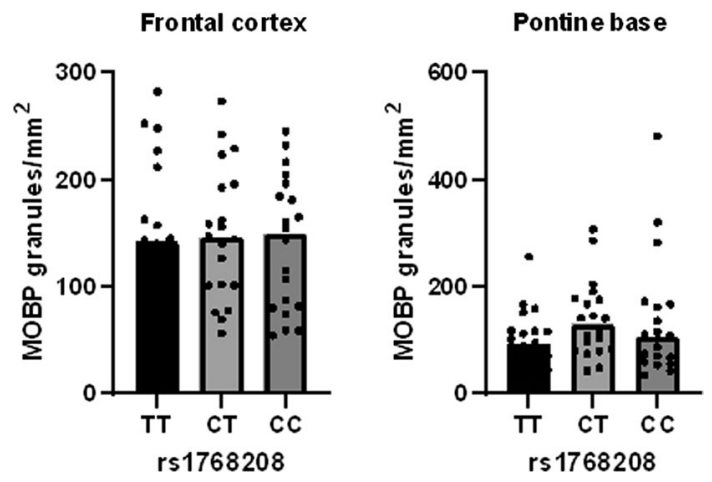

Fig. 5 Quantification of MOBP granular inclusion in PSP. Tissue sections from superior frontal cortex and pons were subjected to MOBP immunohistochemistry, digitally scanned, and analyzed using a custom-designed macro to detect and quantify MOBP granular inclusions. Frontal cortex (left) and pons (right) are stratified by the top PSP GWAS SNP rs1768208 genotype. MOBP, myelin-associated oligodendrocytic basic protein

tested for association with CQN gene expression levels using a linear mixed model implemented with the $\mathrm{R}$ lme 4 package. This identified significant SNP/transcript associations, or eQTLs, at rs3088159 and rs154239 (Table 5). The SNP rs3088159 allele dosage significantly associated with SPTBN5 in temporal cortex $\left(Q=3.22 \times 10^{-4}\right.$, Beta $=-0.212)$ and cerebellum $\left(Q=5.41 \times 10^{-3}\right.$, Beta $=0.237)$, and EHD4 in cerebellum $\left(Q=2.64 \times 10^{-5}\right.$, Beta $=0.231)$. The SNP that was identified to associate with tau threads, rs 154239, showed significant associations with $G H R L$ in temporal cortex $\left(Q=1.91 \times 10^{-3}\right.$, Beta $=-0.203)$ and cerebellum $\left(Q=3.99 \times 10^{-5}\right.$, Beta $=-0.283$ ).

\section{Discussion}

Here we have taken an innovative approach to summarize tau neuropathology in PSP by applying a statistical model principally used in psychometric testing. With the enormous amount of data for 900 cases, 18 brain regions, and 4 brain lesions, this LT modeling was a way to reduce the complex dataset into single, representative values to use as quantitative traits in a GWAS. In doing so, we were able to assign an intermediate phenotype score to each individual PSP case to test for association with their genetic makeup. These LT/SNP associations did not meet the conservative Bonferroni genome-wide significant threshold, but this is most likely due to the small sample size. One may expect to see a strong association with the tau $\mathrm{H} 1 \mathrm{H} 2$ haplotypetagging SNP, rs8072553, however the $\mathrm{H} 2$ allele frequency is too low in PSP to discern a difference in LT/neuropathologic variability.

The LT association analysis results with PSP GWAS top susceptibility loci provide important insight into the methodology employed in this study. For example, the "A" risk allele is associated with lower hindbrain CB burden, but a higher TA hindbrain burden (Fig. 3a,b). Regionally, the positive beta values show that the " $\mathrm{A}$ " risk allele is associated with higher loads of TA lesions in the hindbrain, whereas the rs242557 association with forebrain TAs had negative beta values, suggesting that rs242557 imposes brain regionspecific changes in MAPT expression in PSP. The MAPT locus has been extensively studied [37, 40, 43], and furthermore, there have been large SNP/transcript level associations studies performed for the top PSP GWAS SNPs in human brain tissue $[20,52]$. As we recently reported in a smaller

Table 4 PSP latent trait GWAS results

\begin{tabular}{lcllllllll}
\hline rsID & Chr & Latent trait & Position $^{\mathrm{a}}$ & Alleles & MAF $^{\mathrm{b}}$ & MAF $^{\mathrm{c}}$ & Beta & $P$ value $^{\mathrm{d}}$ & Candidate gene(s) \\
\hline rs3088159 & 15 & NFT & 42190692 & $\mathrm{G}>\mathrm{A}$ & 0.27 & 0.29 & -0.22 & $1.26 \mathrm{E}-06$ & SPTBN5/EHD4 \\
rs154239 & 3 & Threads & 10364793 & $\mathrm{C}>\mathrm{A}$ & 0.37 & 0.35 & -0.20 & $2.78 \mathrm{E}-06$ & SEC13/ATP2B2 \\
rs6762033 & 3 & Overall & 135337113 & $\mathrm{~T}>\mathrm{C}$ & 0.14 & 0.14 & -0.27 & $1.20 \mathrm{E}-05$ & EPHB1/PPP2R3A \\
rs6543017 & 2 & CB & 101698782 & $\mathrm{~A}>\mathrm{G}$ & 0.12 & 0.12 & -0.27 & $1.36 \mathrm{E}-05$ & TBC1D8 \\
rs1333619 & 6 & TA & 137732135 & $\mathrm{G}>\mathrm{A}$ & 0.19 & 0.16 & -0.23 & $1.46 \mathrm{E}-05$ & IFNGR1/OLIG3 \\
rs6771438 & 3 & Overall & 186682324 & $\mathrm{~T}>\mathrm{G}$ & 0.11 & 0.11 & -0.30 & $1.53 \mathrm{E}-05$ & ST6GAL1 \\
rs2305196 & 10 & Threads & 71144324 & $\mathrm{C}>\mathrm{T}$ & 0.28 & 0.25 & -0.19 & $2.19 \mathrm{E}-05$ & HK1 \\
rs16904149 & 8 & NFT & 91229254 & $\mathrm{~T}>\mathrm{G}$ & 0.06 & 0.05 & -0.33 & $7.54 \mathrm{E}-05$ & CALB1/LINCO0534 \\
rs11203544 & 8 & Overall & 13741215 & $\mathrm{C}>\mathrm{T}$ & 0.07 & 0.08 & -0.34 & $8.22 \mathrm{E}-05$ & LOC102725080/SGCZ \\
rs17470661 & 4 & Threads & 45083659 & $\mathrm{G}>\mathrm{A}$ & 0.13 & 0.15 & -0.23 & $9.13 \mathrm{E}-05$ & RP11-362I1.1 \\
\hline
\end{tabular}

$C h r$, chromosome; $M A F$, minor allele frequency; $N F T$, neurofibrillary tangles; $C B$, coiled bodies

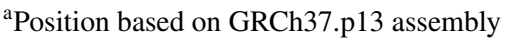

${ }^{\mathrm{b}}$ Minor allele frequency in PSP combined cohort

${ }^{\mathrm{c}}$ Minor allele frequency obtained from 1000 Genomes Project Phase 3 (32) European population (www.1000genomes.org/)

${ }^{\mathrm{d} C}$ Combined analysis $p$ value 
Table 5 Brain transcriptome association with PSP LT GWAS SNPs

\begin{tabular}{|c|c|c|c|c|c|c|c|c|c|}
\hline & Gene & GeneID & SNP & A1 & A2 & BETA & SE & $P$ & $Q$ \\
\hline \multirow[t]{6}{*}{ Temporal cortex } & SPTBN5 & ENSG00000137877 & rs3088159 & $\mathrm{T}$ & $\mathrm{C}$ & -0.212 & 0.042 & $8.51 \mathrm{E}-07$ & $3.22 \mathrm{E}-04$ \\
\hline & GHRL & ENSG00000157017 & rs 154239 & $\mathrm{~T}$ & $\mathrm{G}$ & -0.203 & 0.044 & $6.40 \mathrm{E}-06$ & $1.91 \mathrm{E}-03$ \\
\hline & $R P A P 1$ & ENSG00000103932 & rs3088159 & $\mathrm{T}$ & $\mathrm{C}$ & 0.040 & 0.015 & $7.97 \mathrm{E}-03$ & 0.38 \\
\hline & $P C C B$ & ENSG00000114054 & rs6762033 & $\mathrm{C}$ & $\mathrm{T}$ & -0.069 & 0.030 & $2.49 \mathrm{E}-02$ & 0.58 \\
\hline & $N E C A B 1$ & ENSG00000123119 & rs16904149 & $\mathrm{G}$ & $\mathrm{T}$ & -0.162 & 0.072 & $2.66 \mathrm{E}-02$ & 0.59 \\
\hline & VPS39 & ENSG00000166887 & rs3088159 & $\mathrm{T}$ & $\mathrm{C}$ & -0.033 & 0.015 & $3.15 \mathrm{E}-02$ & 0.62 \\
\hline \multirow[t]{12}{*}{ Cerebellum } & EHD4 & ENSG00000103966 & rs3088159 & $\mathrm{T}$ & $\mathrm{C}$ & 0.231 & 0.041 & $5.97 \mathrm{E}-08$ & $2.64 \mathrm{E}-05$ \\
\hline & $G H R L$ & ENSG00000157017 & rs154239 & $\mathrm{T}$ & G & -0.283 & 0.051 & $9.45 \mathrm{E}-08$ & $3.99 \mathrm{E}-05$ \\
\hline & SPTBN5 & ENSG00000137877 & rs3088159 & $\mathrm{T}$ & $\mathrm{C}$ & 0.237 & 0.055 & $2.51 \mathrm{E}-05$ & $5.41 \mathrm{E}-03$ \\
\hline & $R P A P 1$ & ENSG00000103932 & rs3088159 & $\mathrm{T}$ & $\mathrm{C}$ & 0.045 & 0.015 & $3.69 \mathrm{E}-03$ & 0.23 \\
\hline & ST6GAL1 & ENSG00000073849 & rs6771438 & $\mathrm{G}$ & $\mathrm{T}$ & -0.206 & 0.081 & $1.18 \mathrm{E}-02$ & 0.41 \\
\hline & $M A P K B P 1$ & ENSG00000137802 & rs3088159 & $\mathrm{T}$ & $\mathrm{C}$ & 0.074 & 0.031 & $1.83 \mathrm{E}-02$ & 0.49 \\
\hline & $R P 11-211 G 3.3$ & ENSG00000228804 & rs6771438 & $\mathrm{G}$ & $\mathrm{T}$ & -0.166 & 0.072 & $2.20 \mathrm{E}-02$ & 0.53 \\
\hline & PPA1 & ENSG00000180817 & rs2305196 & $\mathrm{A}$ & $\mathrm{G}$ & 0.060 & 0.026 & $2.45 \mathrm{E}-02$ & 0.55 \\
\hline & CCARI & ENSG00000060339 & rs2305196 & $\mathrm{A}$ & $\mathrm{G}$ & -0.048 & 0.022 & $3.62 \mathrm{E}-02$ & 0.61 \\
\hline & $I L 17 R C$ & ENSG00000163702 & rs 154239 & $\mathrm{~T}$ & G & -0.072 & 0.034 & $3.81 \mathrm{E}-02$ & 0.62 \\
\hline & $R U F Y 2$ & ENSG00000204130 & rs2305196 & $\mathrm{A}$ & G & -0.048 & 0.023 & $4.36 \mathrm{E}-02$ & 0.65 \\
\hline & SEC13 & ENSG00000157020 & rs 154239 & $\mathrm{~T}$ & G & 0.035 & 0.017 & $4.56 \mathrm{E}-02$ & 0.65 \\
\hline
\end{tabular}

SNP, single nucleotide polymorphism; A1, allele 1; A2, allele 2; SE, standard error; Q, FDR adjusted $P$ value

PSP cohort, the most significant associations with rs 242557 and PSP LTs were with glial lesions [3]. The rs242557 H1c $M A P T$ haplotype-tagging SNP is located in a highly conserved regulatory region in $M A P T$ intron 0 . There have been conflicting results using luciferase reporter assays showing that both the " $A$ " risk allele and " $G$ " protective allele can cause an increase luciferase activity [37, 43]. Regardless of these discrepancies, rs242557 does contribute to the regulation of MAPT expression levels, and the LT/rs242557 association shows different effects on lesion type burden comparing CBs to TAs, and in a regional-dependent manner for TAs (Table 2, Fig. 3).

Importantly, these results are in agreement with our current understanding of the H1c sub-haplotype association with the risk of developing PSP. With the identification of rs242557/PSP risk, Rademakers et al. stratified PSP cases by age, and found that PSP patients with an age at death $\leq 75$ years had an increased risk allele frequency (0.62) compared to PSP patients $\geq 76$ at age of death (0.56) [43]. Furthermore, hindbrain-predominant PSP patients are those more likely to die at a younger age compared to the forebrain-predominant patients, due to the greater affection of key brainstem nuclei required for sustaining life relative to higher cortical functions. With our finding that the "A" risk allele is associated with a higher TA burden in the hindbrain, and the increased frequency of the " $\mathrm{A}$ " allele in younger PSP cases, we sought to further understand the relationship between these results. Upon stratifying our PSP cohort into hindbrain- or forebrain-predominant based on available clinical presentations (70\% of the combined cohort, $N=622$ ), consistent differences were identified based on the present knowledge of the H1c sub-haplotype risk and genetic/neuropathologic/clinical features in PSP. The hindbrain PSP patients $(N=77)$ were younger at age of death (mean 73.3 years) compared the forebrain PSP patients $(N=96)$ (mean 77.2 years), and yet typical PSP patients $(N=449)$ had a mean age at death of 73.7 years.

Another PSP risk locus at MOBP (rs 1768208) encodes myelin oligodendrocytic basic protein, a CNS-specific component of myelin, was associated with tau pathology LTs, and more specifically to glial lesion LTs. Based on SNP/ transcript studies in human brain tissue, the " $\mathrm{T}$ " risk allele for rs1768208 is associated with increased MOBP expression levels $[20,52]$. Comparable to our interpretation of rs242557/LT results, the rs1768208/LT associations show that with each copy of the minor allele, there is an increase in tau threads and CB load (i.e. positive beta value).

The novel loci we identified by GWAS may play an important role in PSP based on our current knowledge of SNP/transcript associations for rs242557 and rs1768208 and the neuropathologic associations identified in the present study. The fact that we were able to detect significant SNP/transcript associations with rs3088159 and rs154239 suggest these have a functional role for the genetic associations we identified. Interestingly, Gene Ontology analysis shows that $46 \%(6 / 13)$ of the gene products identified to associate with tau pathology LTs are localized to extracellular exosomes (GO:0070062), which include CALB1, 
EHD4, EPHB1, ST6GAL1, SEC13, and SPTBN5. The SNP rs 1411478 at syntaxin 6 (STX6) was identified in the PSP GWAS as a risk locus, and is involved in vesiclemediated transport in the endocytic and exocytic pathways $[8,50]$. In order to maintain cellular homeostasis, there is a critical interplay of cytoskeletal components including microtubule and actin filaments with motor proteins and membrane-associated vesicle proteins. The loci we identified here in the PSP LT GWAS are enriched in these types of membrane-cytoskeletal proteins along with evidence to support the existence of direct interactions. For example, spectrin beta chain, non-erythrocytic 5 (SPTBN5) and tau are both cytoskeletal proteins and it has been reported that tau inhibits F-actin crosslinking activity of spectrin [9]. More recently, Krieg et al. showed that the combination of spectrin and microtubules provide axonal and dendritic protection from mechanical stress in C. elegans with mutations in $\beta$-spectrin and tau homologues [28]. Additionally, SPTBN2 mutations have been identified to cause spinocerebellar ataxia type 5 via a mechanism involving glutamate receptors, setting precedence for mutant spectrin protein to cause neurodegeneration [21]. The SNP/transcript association at rs3088159 was also associated with EHD4 transcript levels (Table 5). Eps15 Homology Domain Protein (EHD) family protein 4, or EHD4, was originally identified as a extracellular matrix protein [29] and found later to play an important role in endosomal recycling and is involved in the control of trafficking at the early endosome, regulating exit of cargo toward both the recycling compartment and the late endocytic pathway [47]. Taken together, our results suggest that extracellular exosomes play an important role in PSP disease pathogenesis.

Some of the novel SNP/LT loci are known to be involved in Alzheimer's disease (AD), neuronal health, and tau phosphorylation. Calbindin (CALB1) is a calcium-binding protein reported to be involved in $\mathrm{AD}$, and plays an important role in preventing neuronal death [17, 23]. Protein phosphatase 2 regulatory subunit B" alpha (PPP2R3A) is one of the subunits of protein phosphatase 2, which as a major Ser/Thr phosphatase, has been extensively studied in the context of AD and tau phosphorylation homeostasis [22]. The tyrosine kinase ephrin B1 (EPHB1) is also interesting candidate gene that may be involved in PSP pathogenesis. The interplay between EPHB1, EPHB2, and GSK-3 $\beta$ plays a significant role in regulating tau phosphorylation levels [24].

In conclusion, using tau neuropathologic heterogeneity as intermediate phenotypes in PSP is a novel approach to identifying QTLs. Testing for genetic association with a biological measurement within a disease cohort, rather than a case-control genetic association study, may facilitate the identification of biological mechanisms underlying disease pathogenesis.
Supplementary Information The online version contains supplementary material available at https://doi.org/10.1007/s00401-021-02289-0.

Acknowledgements We thank the patients and their families for participating in and contributing to these research studies. DWD was supported by NIH awards: UG3 NS104095, R01 AG054449, U54 NS100693, P30 AG062677, as well as the Rainwater Charitable Foundation and the Robert E. Jacoby Professorship. OAR was supported in part by NIH awards: R01 NS78086, U54-NS100693, R01 AG56366, the US Department of Defense (W81XWH-17-1-0249), and American Parkinson Disease Association (APDA) Center for Advanced Research. ZKW is partially supported by the Mayo Clinic Center for Regenerative Medicine, the gifts from The Sol Goldman Charitable Trust, and the Donald G. and Jodi P. Heeringa Family, the Haworth Family Professorship in Neurodegenerative Diseases fund, and The Albertson Parkinson's Research Foundation. He serves as PI or Co-PI on Biogen, Inc. (228PD201), Biohaven Pharmaceuticals, Inc. (BHV4157-206 and BHV3241-301), and Neuraly, Inc. (NLY01-PD-1) grants. He serves as Co-PI of the Mayo Clinic APDA Center for Advanced Research. GDS was supported by awards: P01 AG017586, U54 NS100693, and UG3 NS104095.

Open Access This article is licensed under a Creative Commons Attribution 4.0 International License, which permits use, sharing, adaptation, distribution and reproduction in any medium or format, as long as you give appropriate credit to the original author(s) and the source, provide a link to the Creative Commons licence, and indicate if changes were made. The images or other third party material in this article are included in the article's Creative Commons licence, unless indicated otherwise in a credit line to the material. If material is not included in the article's Creative Commons licence and your intended use is not permitted by statutory regulation or exceeds the permitted use, you will need to obtain permission directly from the copyright holder. To view a copy of this licence, visit http://creativecommons.org/licenses/by/4.0/.

\section{References}

1. Ahmed Z, Josephs KA, Gonzalez J, DelleDonne A, Dickson DW (2008) Clinical and neuropathologic features of progressive supranuclear palsy with severe pallido-nigro-luysial degeneration and axonal dystrophy. Brain 131:460-472. https://doi.org/10.1093/ brain/awm301

2. Allen M, Carrasquillo MM, Funk C, Heavner BD, Zou F, Younkin CS et al (2016) Human whole genome genotype and transcriptome data for Alzheimer's and other neurodegenerative diseases. Sci Data 3:160089. https://doi.org/10.1038/sdata.2016.89

3. Allen M, Burgess JD, Ballard T, Serie D, Wang X, Younkin CS et al (2016) Gene expression, methylation and neuropathology correlations at progressive supranuclear palsy risk loci. Acta Neuropathol 132:197-211. https://doi.org/10.1007/s0040 1-016-1576-7

4. Allen M, Wang X, Burgess JD, Watzlawik J, Serie DJ, Younkin CS et al (2018) Conserved brain myelination networks are altered in Alzheimer's and other neurodegenerative diseases. Alzheimers Dement 14:352-366. https://doi.org/10.1016/j.jalz.2017.09.012

5. Arima K, Nakamura M, Sunohara N, Ogawa M, Anno M, Izumiyama Y et al (1997) Ultrastructural characterization of the tauimmunoreactive tubules in the oligodendroglial perikarya and their inner loop processes in progressive supranuclear palsy. Acta Neuropathol 93:558-566. https://doi.org/10.1007/s004010050652

6. Baker M, Litvan I, Houlden H, Adamson J, Dickson D, Perez-Tur J et al (1999) Association of an extended haplotype in the tau gene 
with progressive supranuclear palsy. Hum Mol Genet 8:711-715. https://doi.org/10.1093/hmg/8.4.711

7. Bates D, Machler M, Bolker BM, Walker SC (2015) Fitting Linear Mixed-Effects Models Using lme4. J Stat Softw 67: 1-48. https:// doi.org/10.18637/jss.v067.i01

8. Bock JB, Lin RC, Scheller RH (1996) A new syntaxin family member implicated in targeting of intracellular transport vesicles. J Biol Chem 271:17961-17965. https://doi.org/10.1074/ jbc.271.30.17961

9. Carlier MF, Simon C, Cassoly R, Pradel LA (1984) Interaction between microtubule-associated protein tau and spectrin. Biochimie 66:305-311. https://doi.org/10.1016/0300-9084(84)90007 $-5$

10. Chang CC, Chow CC, Tellier LC, Vattikuti S, Purcell SM, Lee JJ (2015) Second-generation PLINK: rising to the challenge of larger and richer datasets. Gigascience 4:7. https://doi. org/10.1186/s13742-015-0047-8

11. Chen JA, Chen Z, Won H, Huang AY, Lowe JK, Wojta K et al (2018) Joint genome-wide association study of progressive supranuclear palsy identifies novel susceptibility loci and genetic correlation to neurodegenerative diseases. Mol Neurodegener 13:41. https://doi.org/10.1186/s13024-018-0270-8

12. Conrad C, Andreadis A, Trojanowski JQ, Dickson DW, Kang $D$, Chen X et al (1997) Genetic evidence for the involvement of tau in progressive supranuclear palsy. Ann Neurol 41:277-281. https://doi.org/10.1002/ana.410410222

13. Das S, Forer L, Schonherr S, Sidore C, Locke AE, Kwong A et al (2016) Next-generation genotype imputation service and methods. Nat Genet 48:1284-1287. https://doi.org/10.1038/ ng. 3656

14. Dickson DW, Ahmed Z, Algom AA, Tsuboi Y, Josephs KA (2010) Neuropathology of variants of progressive supranuclear palsy. Curr Opin Neurol 23:394-400. https://doi.org/10.1097/ WCO.0b013e32833be924

15. Evans W, Fung HC, Steele J, Eerola J, Tienari P, Pittman A et al (2004) The tau H2 haplotype is almost exclusively Caucasian in origin. Neurosci Lett 369:183-185. https://doi.org/10.1016/j.neule t.2004.05.119

16. Fujioka S, Sanchez Contreras MY, Strongosky AJ, Ogaki K, Whaley NR, Tacik PM et al (2015) Three sib-pairs of autopsy-confirmed progressive supranuclear palsy. Parkinsonism Relat Disord 21:101-105. https://doi.org/10.1016/j.parkreldis.2014.10.028

17. Geula C, Nagykery N, Wu CK, Bu J (2003) Loss of calbindinD28K from aging human cholinergic basal forebrain: relation to plaques and tangles. J Neuropathol Exp Neurol 62:605-616. https ://doi.org/10.1093/jnen/62.6.605

18. Hansen KD, Irizarry RA, Wu Z (2012) Removing technical variability in RNA-seq data using conditional quantile normalization. Biostatistics 13:204-216. https://doi.org/10.1093/biostatist ics/kxr054

19. Hauw JJ, Daniel SE, Dickson D, Horoupian DS, Jellinger K, Lantos PL et al (1994) Preliminary NINDS neuropathologic criteria for Steele-Richardson-Olszewski syndrome (progressive supranuclear palsy). Neurology 44:2015-2019. https://doi.org/10.1212/ wnl.44.11.2015

20. Hoglinger GU, Melhem NM, Dickson DW, Sleiman PM, Wang LS, Klei L et al (2011) Identification of common variants influencing risk of the tauopathy progressive supranuclear palsy. Nat Genet 43:699-705. https://doi.org/10.1038/ng.859

21. Ikeda Y, Dick KA, Weatherspoon MR, Gincel D, Armbrust KR, Dalton JC et al (2006) Spectrin mutations cause spinocerebellar ataxia type 5. Nat Genet 38:184-190. https://doi.org/10.1038/ ng 1728

22. Iqbal K, Liu F, Gong CX, Alonso Adel C, Grundke-Iqbal I (2009) Mechanisms of tau-induced neurodegeneration. Acta Neuropathol 118:53-69. https://doi.org/10.1007/s00401-009-0486-3
23. Iritani S, Niizato K, Emson PC (2001) Relationship of calbindin D28K-immunoreactive cells and neuropathological changes in the hippocampal formation of Alzheimer's disease. Neuropathology 21:162-167

24. Jiang J, Wang ZH, Qu M, Gao D, Liu XP, Zhu LQ et al (2015) Stimulation of EphB2 attenuates tau phosphorylation through PI3K/Akt-mediated inactivation of glycogen synthase kinase3beta. Sci Rep 5:11765. https://doi.org/10.1038/srep11765

25. Jun G, Flickinger M, Hetrick KN, Romm JM, Doheny KF, Abecasis GR et al (2012) Detecting and estimating contamination of human DNA samples in sequencing and array-based genotype data. Am J Hum Genet 91:839-848. https://doi.org/10.1016/j. ajhg.2012.09.004

26. Kent WJ (2002) BLAT-the BLAST-like alignment tool. Genome Res 12:656-664. https://doi.org/10.1101/gr.229202

27. Kenward MG, Roger JH (1997) Small sample inference for fixed effects from restricted maximum likelihood. Biometrics 53:983-997

28. Krieg M, Stuhmer J, Cueva JG, Fetter R, Spilker K, Cremers D, et al (2017) Genetic defects in beta-spectrin and tau sensitize C. elegans axons to movement-induced damage via torque-tension coupling. Elife. https://doi.org/10.7554/eLife.20172

29. Kuo HJ, Tran NT, Clary SA, Morris NP, Glanville RW (2001) Characterization of EHD4, an EH domain-containing protein expressed in the extracellular matrix. J Biol Chem 276:4310343110. https://doi.org/10.1074/jbc.M106128200

30. Kuznetsova A, Brockhoff PB, Christensen RHB (2017) lmertest package: tests in linear mixed effects models. J Stat Softw 82: 1-26. https://doi.org/10.18637/jss.v082.i13

31. Litvan I, Hauw JJ, Bartko JJ, Lantos PL, Daniel SE, Horoupian DS et al (1996) Validity and reliability of the preliminary NINDS neuropathologic criteria for progressive supranuclear palsy and related disorders. J Neuropathol Exp Neurol 55:97-105. https:// doi.org/10.1097/00005072-199601000-00010

32. Loh PR, Danecek P, Palamara PF, Fuchsberger C, Y AR, H KF, et al (2016) Reference-based phasing using the Haplotype Reference Consortium panel. Nat Genet 48: 1443-1448https://doi. org/10.1038/ng.3679

33. Manichaikul A, Mychaleckyj JC, Rich SS, Daly K, Sale M, Chen WM (2010) Robust relationship inference in genome-wide association studies. Bioinformatics 26:2867-2873. https://doi. org/10.1093/bioinformatics/btq559

34. Matsusaka H, Ikeda K, Akiyama H, Arai T, Inoue M, Yagishita S (1998) Astrocytic pathology in progressive supranuclear palsy: significance for neuropathological diagnosis. Acta Neuropathol 96:248-252. https://doi.org/10.1007/s004010050891

35. McCarthy S, Das S, Kretzschmar W, Delaneau O, Wood AR, Teumer A et al (2016) A reference panel of 64,976 haplotypes for genotype imputation. Nat Genet 48:1279-1283. https://doi. org/10.1038/ng.3643

36. Mi H, Poudel S, Muruganujan A, Casagrande JT, Thomas PD (2016) PANTHER version 10: expanded protein families and functions, and analysis tools. Nucleic Acids Res 44:D336-342. https://doi.org/10.1093/nar/gkv1194

37. Myers AJ, Pittman AM, Zhao AS, Rohrer K, Kaleem M, Marlowe L et al (2007) The MAPT H1c risk haplotype is associated with increased expression of tau and especially of 4 repeat containing transcripts. Neurobiol Dis 25:561-570. https://doi.org/10.1016/j. nbd.2006.10.018

38. Nishimura M, Namba Y, Ikeda K, Oda M (1992) Glial fibrillary tangles with straight tubules in the brains of patients with progressive supranuclear palsy. Neurosci Lett 143:35-38. https://doi. org/10.1016/0304-3940(92)90227-x

39. Patterson N, Price AL, Reich D (2006) Population structure and eigenanalysis. PLoS Genet 2:e190. https://doi.org/10.1371/journ al.pgen.0020190 
40. Pittman AM, Myers AJ, Abou-Sleiman P, Fung HC, Kaleem M, Marlowe L et al (2005) Linkage disequilibrium fine mapping and haplotype association analysis of the tau gene in progressive supranuclear palsy and corticobasal degeneration. J Med Genet 42:837-846. https://doi.org/10.1136/jmg.2005.031377

41. Price AL, Patterson NJ, Plenge RM, Weinblatt ME, Shadick NA, Reich D (2006) Principal components analysis corrects for stratification in genome-wide association studies. Nat Genet 38:904909. https://doi.org/10.1038/ng 1847

42. Purcell S, Neale B, Todd-Brown K, Thomas L, Ferreira MA, Bender D et al (2007) PLINK: a tool set for whole-genome association and population-based linkage analyses. Am J Hum Genet 81:559-575. https://doi.org/10.1086/519795

43. Rademakers R, Melquist S, Cruts M, Theuns J, Del-Favero J, Poorkaj P et al (2005) High-density SNP haplotyping suggests altered regulation of tau gene expression in progressive supranuclear palsy. Hum Mol Genet 14:3281-3292. https://doi. org $/ 10.1093 / \mathrm{hmg} / \mathrm{ddi} 361$

44. Rizopoulos D (2006) ltm: an R package for latent variable modelling and Item Response Theory analyses. J Stat Softw 17: 1-25. https://doi.org/10.18637/jss.v017.i05

45. Samejima F (1969) Estimation of latent ability using a response pattern of graded scores. Psychometrika Monograph Supplement, City

46. Sanchez-Contreras MY, Kouri N, Cook CN, Serie DJ, Heckman MG, Finch NA et al (2018) Replication of progressive supranuclear palsy genome-wide association study identifies SLCO1A2 and DUSP10 as new susceptibility loci. Mol Neurodegener 13:37. https://doi.org/10.1186/s13024-018-0267-3

47. Sharma M, Naslavsky N, Caplan S (2008) A role for EHD4 in the regulation of early endosomal transport. Traffic 9:995-1018. https ://doi.org/10.1111/j.1600-0854.2008.00732.x
48. Van der Auwera GA, Carneiro MO, Hartl C, Poplin R, Del Angel G, Levy-Moonshine A, et al (2013) From FastQ data to high confidence variant calls: the Genome Analysis Toolkit best practices pipeline. Curr Protoc Bioinform 43: 111011-111033. https://doi. org/10.1002/0471250953.bi1110s43

49. Wang K, Li M, Hakonarson H (2010) ANNOVAR: functional annotation of genetic variants from high-throughput sequencing data. Nucleic Acids Res 38:e164. https://doi.org/10.1093/nar/ gkq603

50. Wendler F, Tooze $S$ (2001) Syntaxin 6: the promiscuous behaviour of a SNARE protein. Traffic 2: 606-611. https://doi.org/10.103 4/j.1600-0854.2001.20903.x

51. Williams DR, de Silva R, Paviour DC (2005) Characteristics of two distinct clinical phenotypes in pathologically proven progressive supranuclear palsy: Richardson's syndrome and PSPparkinsonism. Brain 128:1247-1258. https://doi.org/10.1093/ brain/awh488

52. Zou F, Chai HS, Younkin CS, Allen M, Crook J, Pankratz VS et al (2012) Brain expression genome-wide association study (eGWAS) identifies human disease-associated variants. PLoS Genet 8:e1002707. https://doi.org/10.1371/journal.pgen.1002707

Publisher's Note Springer Nature remains neutral with regard to jurisdictional claims in published maps and institutional affiliations. 